\title{
Addendum to: \\ A Bound for the Fixed-Point Index \\ of an Area-Preserving Map \\ with Applications to Mechanics
}

Carl P. Simon (Ann Arbor)

Theorems 1 and 2 in the first half of Simon [3] have been proven independently by N.Nikishin [2]. They had previously been formulated as a hypothesis by A. I. Shnirelman (see [1]) and others.

\section{References}

1. Arnold, V.: An appendix to the Russian translation of Poincarés works, p. 988. Moscow 1972

2. Nikishin, N.: Fixed points of diffeomorphisms on the two-sphere that preserve area. Funkcional Anal. i Prelozen 8, 84-85 (1974)

3. Simon, C.P.: A bound for the fixed point index of an area-preserving map with applications to mechanics. Inventiones math. 26, 187-200(1974)

Received August 16, 1975

Carl P. Simon

The University of Michigan

Department of Mathematics

Ann Arbor, Michigan 48104

USA 